\title{
Influence of eccentric cyclic loading on implant components: Comparison between titanium and zirconia abutments
}

\author{
Ryotaro NAKANO, Shinya HOMMA, Takuya TAKANASHI, Tomoki HIRANO, Yoshitaka FURUYA \\ and Yasutomo YAJIMA \\ Department of Oral and Maxillofacial Implantology, Tokyo Dental College, 2-9-18 Kandamisaki-cho, Chiyoda-ku, Tokyo 101-0061, Japan \\ Corresponding author, Ryotaro NAKANO; E-mail: rnakano@tdc.ac.jp
}

\begin{abstract}
The objective of this study was to investigate the effects of eccentric cyclic loading on implant components using the internal joint system with titanium and zirconia abutments. Abutments were made of either pure titanium (Ti group) or zirconia (TZP group). Cyclic loading test was conducted according to the specifications of ISO 14801. Loading condition was at 2 points assuming axial load and eccentric load. The reverse torque value reduced after the eccentric load and reduced more in the TZP group than the Ti group. Marginal gap changed after eccentric loading, and was greater in the TZP group. In the TZP group, changes in configuration were observed in the implant body, and Ti was detected on the abutment surface. From the above, the eccentric load may have worse effects than axial loads. It was suggested that the TZP group was clinically disadvantageous compared to the Ti group.
\end{abstract}

Keywords: Zirconia, Titanium, Implant abutment, Eccentric moment, Fatigue test

\section{INTRODUCTION}

Occlusal force includes axial loads in the direction of the tooth axis and eccentric loads in directions deviating from the tooth axis. Eccentric loads have a greater influence on implant components compared to axial loads ${ }^{1,2)}$. Reports have indicated that this effect may cause complications after implant treatment such as screw loosening, overloading on the surrounding bone, and misfit at the implant-abutment interface ${ }^{2-4)}$.

Titanium abutments are currently used as a standard for implant treatment ${ }^{5,6}$. However, various complications, such as discoloration of the peri-implant mucosa or metal color exposure due to gum recession, may occur when titanium abutments are used in esthetic regions such as the anterior teeth $^{6-8}$. In contrast to titanium abutments, zirconia abutments not only have better esthetic features such as natural tooth like color and high translucency, but also possess exceptional tissue-compatibility and fracture strength ${ }^{7,9-11)}$. For these reasons, the application of zirconia abutments in the anterior region has been increasing ${ }^{12)}$. Since the long axis of the implant assembly placed in the anterior region is inclined with respect to the occlusal plane, implants is susceptible to eccentric loading ${ }^{13)}$. Therefore, it is necessary to investigate the effects of eccentric loading on implant components with zirconia abutments.

There are many reports on the effects of cyclic loading on implant components using the external joint system with zirconia abutments ${ }^{6,7,14-18)}$; however, a very few studies used the internal joint system $^{19-21)}$. The paper that observed the inner surface of the implant body evaluated implant components with titanium and zirconia abutments only under an eccentric load ${ }^{19)}$.

Color figures can be viewed in the online issue, which is available at J-STAGE.

Received Jan 28, 2020: Accepted Apr 6, 2020

doi:10.4012/dmj.2020-030 JOI JST.JSTAGE/dmj/2020-030
The papers that investigated the abutment screw and analyzed the fracture surface after the fatigue test evaluated implant components with zirconia abutments only under an axial load ${ }^{20,21)}$. In other words, there has only been a single report comparing implant components using the internal joint system with titanium and zirconia abutments ${ }^{19)}$. In addition, no studies have considered the mechanical aspects of different loads (axial and eccentric load) on implant components using the internal joint system with zirconia abutments. The objective of this study was to investigate the effects of eccentric cyclic loading on implant components using the internal joint system with titanium and zirconia abutments in order to identify the differences.

\section{MATERIALS AND METHODS}

The implant body made of pure titanium (cp-Ti: grade 4) and the internal joint system with butt joint connection was used in this experiment $(\Phi 4.4 \times 12 \mathrm{~mm}$, GC implant Re, GC, Tokyo, Japan) (Fig. 1). The abutment materials included cp-Ti (grade 4) and zirconia (yttria-stabilized tetragonal zirconia polycrystal: Y-TZP, Aadva, GC) (Fig. 2). The abutment screw was made of a titanium alloy (Ti$6 \mathrm{Al}-4 \mathrm{~V})$ specified by the manufacturer. Specimens with titanium abutment will be referred to as the Ti group and specimens with zirconia abutment will be referred to as the TZP group.

\section{Cyclic loading test}

Cyclic loading test was conducted according to ISO $14801^{22)}$. The implant specimen and testing jig are shown in Fig. 3. A stainless-steel superstructure was fabricated via CAD/CAM (M32-V, CITIZEN MACHINERY, Nagano, Japan) and was attached to the abutment on the implant body for testing. The implant 


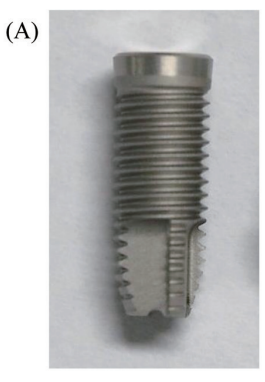

(B)

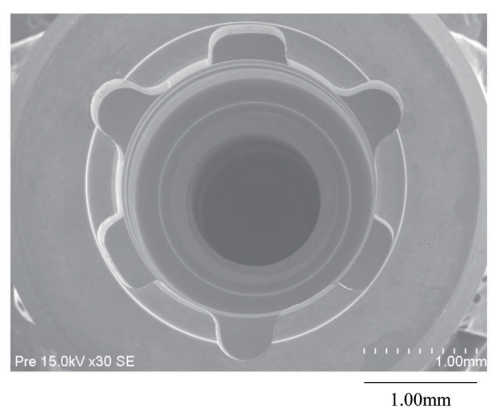

(A)
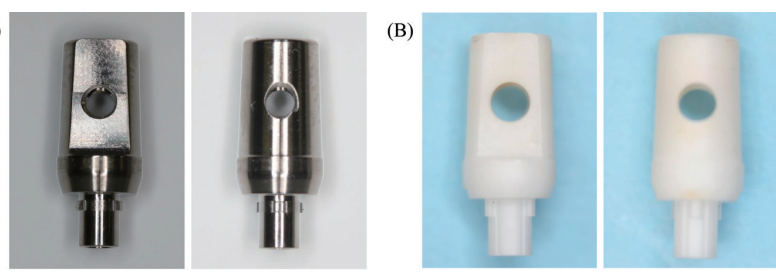

Fig. 2 (A) Pure titanium (grade 4) abutment and (B) Zirconia (Y-TZP) abutment.

Fig. 1 Implant body used in this study.

(A) Overview, (B) Top view.

(A)

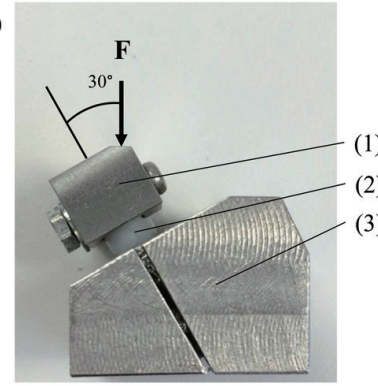

(1) Suprastructure for testing

(2) Implant specimen

(3) Specimen holder
(B)

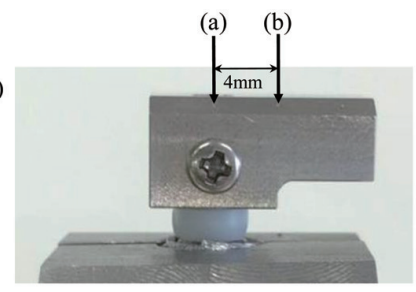

Loading condition

(a) Axial load: Point directly above the implant body

(b) Eccentric load: Point $4 \mathrm{~mm}$ deviated from the axial load point

Fig. 3 Implant specimen and testing jig.

(A) side view and (B) frontal view of suprastructure for testing (Implant assembly was fixed in a specimen holder at $30^{\circ}$ to the vertical axis.).

Table 1 Abutment materials and loading conditions

\begin{tabular}{|c|c|c|c|}
\hline Abutment materials & \multicolumn{2}{|c|}{ Loading conditions } & Abbreviation \\
\hline \multirow{3}{*}{ Ti Group } & Without loading & & Ti-pre \\
\hline & Above implant body & Axial load & $\mathrm{Ti}-0 \mathrm{~mm}$ \\
\hline & $4 \mathrm{~mm}$ deviated & Eccentric load & $\mathrm{Ti}-4 \mathrm{~mm}$ \\
\hline \multirow{3}{*}{ TZP Group } & Without loading & & TZP-pre \\
\hline & Above implant body & Axial load & TZP-0mm \\
\hline & $4 \mathrm{~mm}$ deviated & Eccentric load & TZP-4mm \\
\hline
\end{tabular}

assembly was fixed in a specimen holder at an angle of $30^{\circ}$ to the vertical axis (Fig. 3A). Loading conditions was set at 2 points: a point directly above the implant body assuming axial load, and a point $4 \mathrm{~mm}$ deviated from the axial load point assuming eccentric load (Fig. 3B).

A servo-driven load cell type testing machine (TY-100, Techno Ark, Nagano, Japan) was used for performing the cyclic fatigue test. Test conditions were established in distilled water at $37^{\circ} \mathrm{C}$ with load of 300 $\mathrm{N}$, load cycles of $1 \times 10^{6}$, and frequency of $2 \mathrm{~Hz}$. Four categories were made according to abutment material and load condition, and compared before and after loading (Table 1). Five specimens were prepared for each group.

\section{Experimental protocol}

The experimental protocol is shown in Fig. 4. Measurements of reverse torque and marginal gap, and surface observation of implant components were performed before and after cyclic loading test for the Ti and TZP groups. For the TZP group, Quantitative evaluation of changes in configuration was performed on the implant and elemental analysis of the abutment was performed after the cyclic loading test. Changes in configuration of the implant body and elemental analysis of the abutment for the Ti group were not measured, because no change in configuration was observed in both the implant body and abutment. 


\section{Measurement of reverse torque}

The reverse torque value was measured by referring to past reports ${ }^{2,23,24)}$. A digital torque meter (BTG50CN, Tonichi, Tokyo, Japan) was used for measuring the reverse torque value and tightening of the abutment screw (Fig. 5A). First, the abutment screw was tightened with the manufacturer recommended torque value ( $\mathrm{Ti}$ group: $20 \mathrm{Ncm}$, TZP group: $30 \mathrm{Ncm}$ ) and left for $10 \mathrm{~min}$. Subsequently, the abutment screw was re-tightened with the manufacturer recommended torque value and left for $5 \mathrm{~min}$, and then the reverse torque value before cyclic loading test was measured. After measuring the reverse torque value, the abutment screw was tightened again with the manufacturer's recommended torque value before test; 10 min later, the abutment screw was re-tightened to the same torque to prevent loosening of

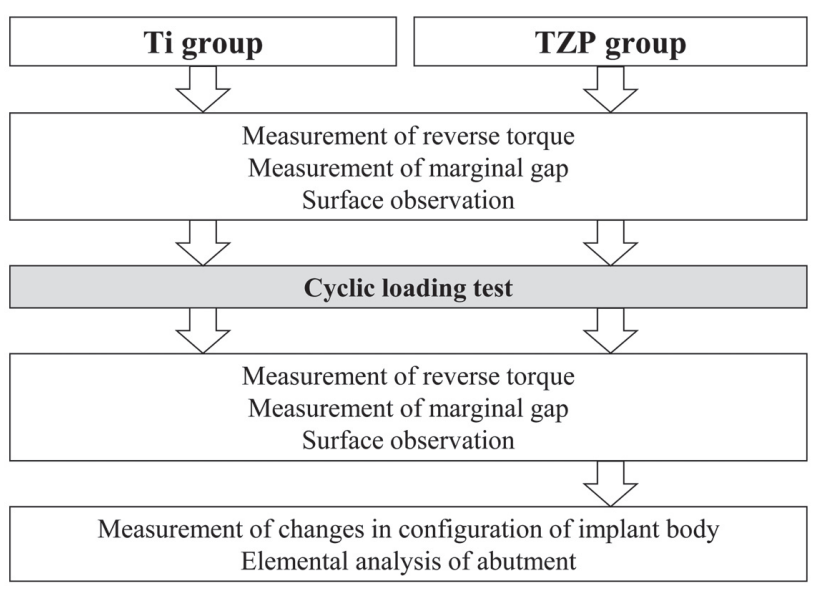

Fig. 4 Experimental protocol. the initial torque and minimize the influence of settling. After test, the reverse torque value was measured again (Fig. 5B). The values reduced from the tightening torque value to the reverse torque before test was compared between the groups. The reverse torque values before and after test were compared within the same groups and under the same loading conditions. In addition, the values reduced from before to after test were compared between the groups after the same loading conditions. Since the tightening torque value was different between groups, the reduction values were used for comparisons between the groups.

\section{Measurement of marginal gap}

The marginal gap was measured by referring to past report ${ }^{18)}$. The gap was captured at a magnification of 5,000 using a scanning electron microscope (SEM; SU6600, Hitachi, Tokyo, Japan) from a direction perpendicular to the long axis of the implant using the same platform, and the distance between the bottom edge of each abutment and the top edge of the implant was measured using a measuring tool in the SEM. Five specimens were measured before and after the test for each condition (Ti-0mm, Ti-4mm, TZP-0mm, TZP-4mm); SEM images were taken on 4 points rotated at $90^{\circ}$, which were considered regions of interest (ROI) (Fig. 6A), and 3 points (a, b, c) that were equally within the ROI were established as measurement points (Fig. 6B). Each point was measured 3 times for a total of 36 measurements for each specimen. The average value was used as the marginal gap value. The marginal gaps were compared between the groups under the same loading conditions and between loading conditions within the same groups.
(A)

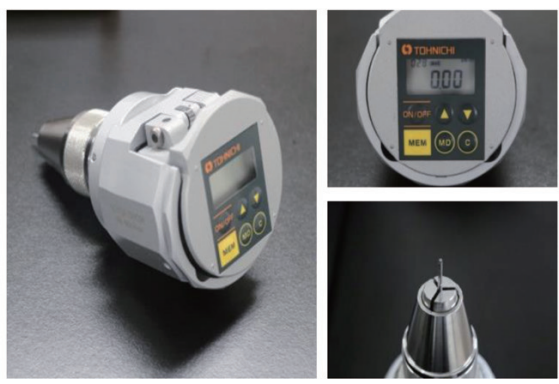

(B)

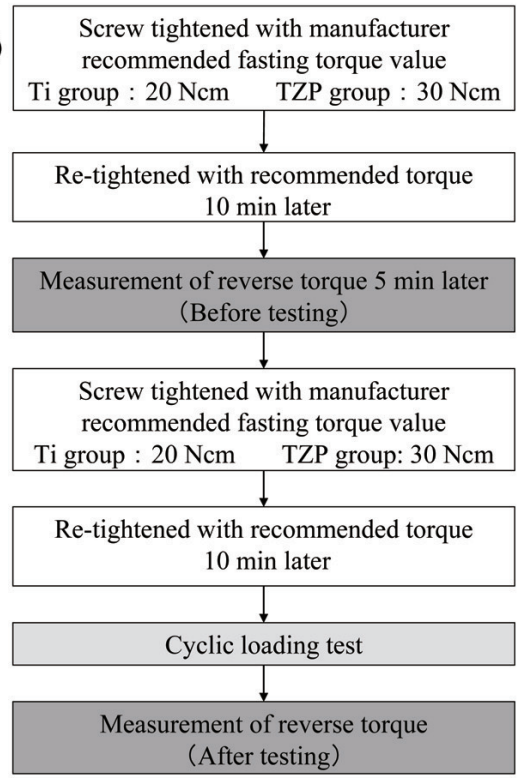

Fig. 5 Measurement of reverse torque.

(A) Digital torque meter. (B) Procedure for measuring reverse torque. 


\section{Surface observation}

Implant body and abutment surfaces were observed. SEM images were captured at an angle of $60^{\circ}$ against longitudinal axis of the implant (Fig. 7A). In addition, the abutment was observed on the direction perpendicular to the long axis of the abutment using SEM (Figs. 7B and $\mathrm{C}$ ). The side of anti-rotation was used as the ROI, and the surface of the ROI was observed using SEM.

Change in configuration of TZP group implant body Quantitative evaluation of change in configuration was performed on the implant body of the TZP group. The site of changes in configuration that occurred on the anti-
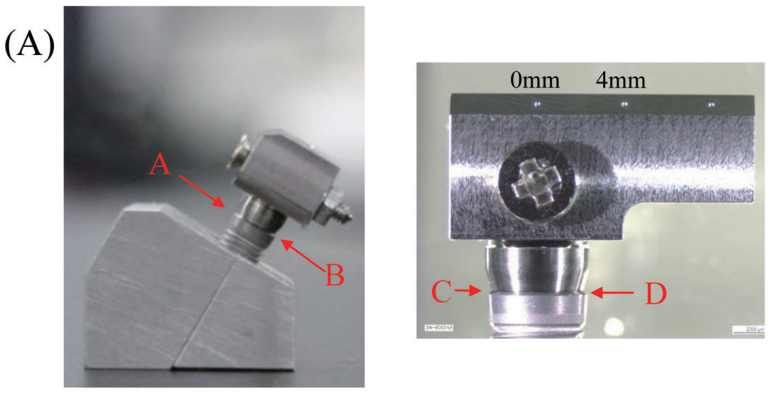

(B)

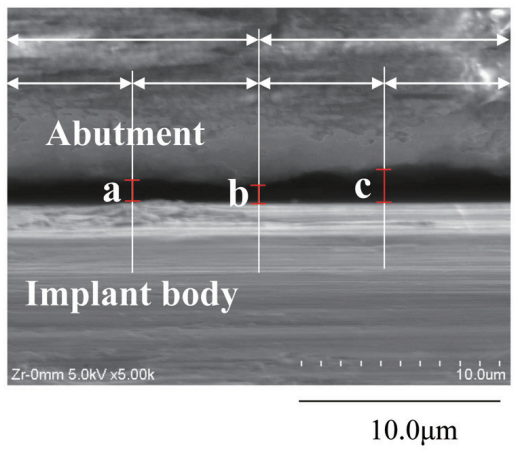

Fig. 6 Method of measuring marginal gap.

(A) Direction for taking the SEM images (A-D). (B) Measurement points within ROI. rotation of implant body was captured at a magnification of 400 using SEM and was used as the ROI (Fig. 8B). The region of changes in configuration was selected using a graphic editing program (Adobe Photoshop CS 6), and the pixel number was measured (Fig. 8C). At the same time, the number of pixels of SEM observation region
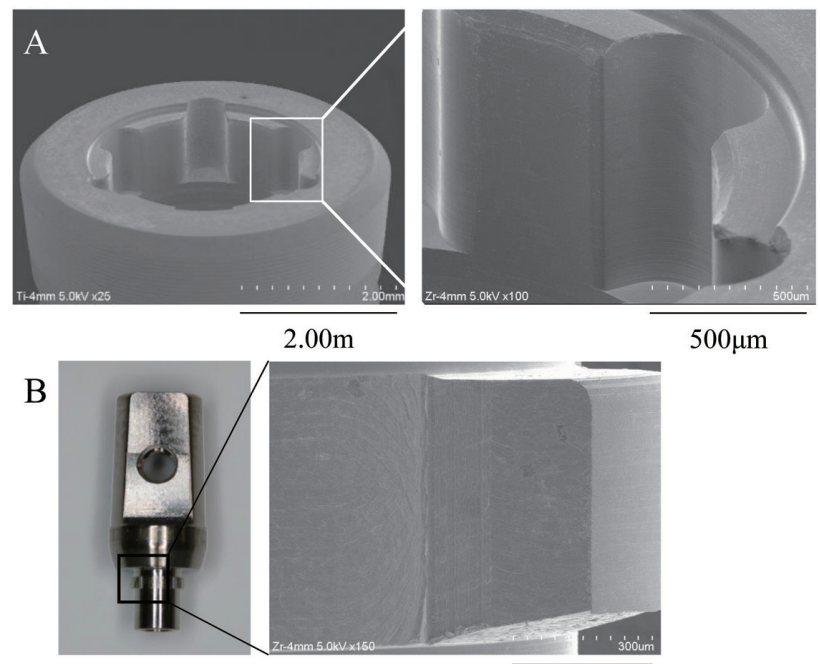

$2.00 \mathrm{~m}$ $500 \mu \mathrm{m}$
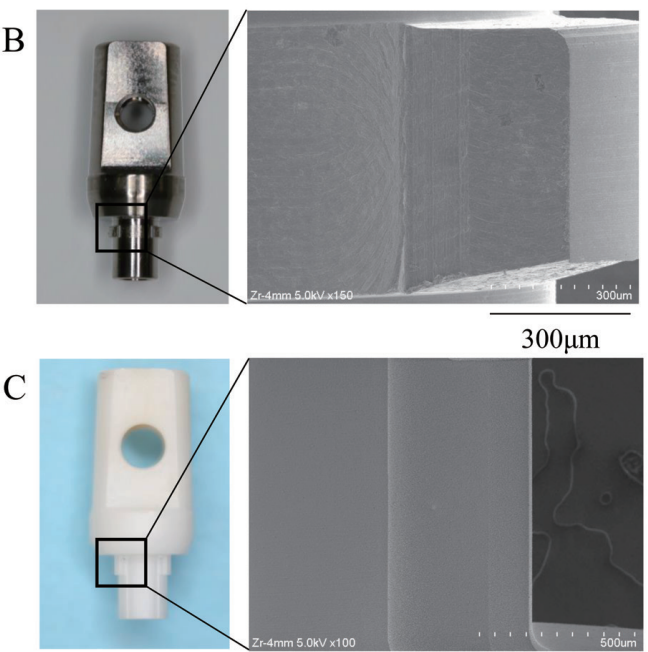

$300 \mu \mathrm{m}$

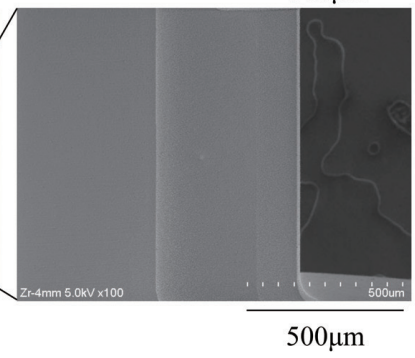

Fig. 7 Surface observation using SEM.

(A)Anti-rotation on implant body before loading(captured at an angle of $60^{\circ}$ ). (B)Antirotation on $\mathrm{Ti}$ abutment before loading (captured horizontally). (C)Anti-rotation on TZP abutment before loading (captured horizontally).
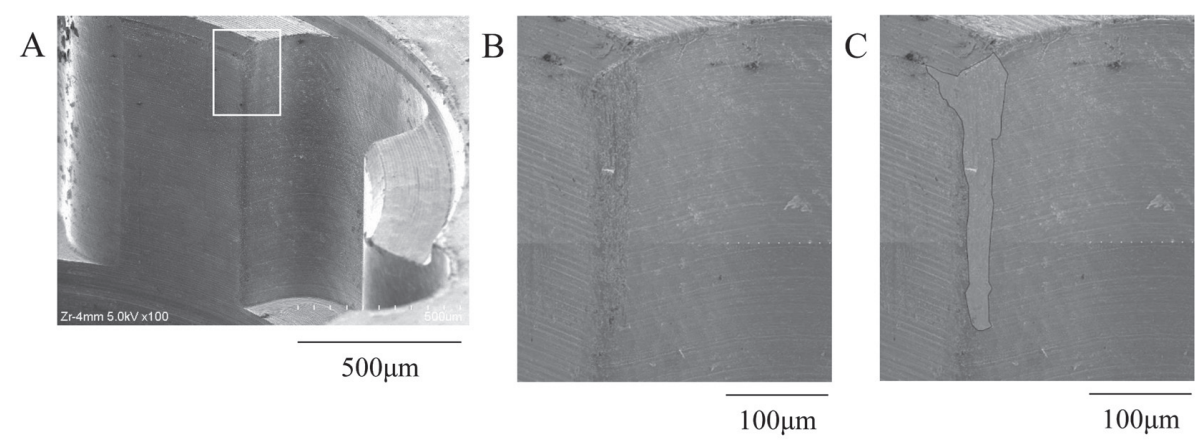

Fig. 8 Evaluation sites of changes in configuration on implant body in TZP group. (A) SEM image of implant anti-rotation of TZP group and (B) Top of the implant body anti-rotation where configurational change was observed, and (C) during selection of sites of configurational change. 
$100 \times 100 \mu \mathrm{m}$ was measured and the area of changes in configuration was calculated from the number of pixels. The 3 anti-rotations for one specimen were measured three times each. Since the number of specimens was 5 , the total measurement points were 45 , and the average value was calculated. The load condition (TZP-0mm, $\mathrm{TZP}-4 \mathrm{~mm}$ ) was compared by the average value.

\section{Elemental analysis of TZP group}

In the anti-rotation of abutment in the TZP group, elemental analysis was performed after the cyclic loading test using the electron probe micro analyzer (EPMA; JXA - 8200, JEOL, Tokyo, Japan).

\section{Statistical analysis}

The marginal gap comparing the influence of loading conditions within the same groups were subjected to Tukey's multiple comparison test after one-way analysis of variance (ANOVA). The reverse torque in all comparisons, the marginal gap comparing the influence between the groups under the same loading conditions
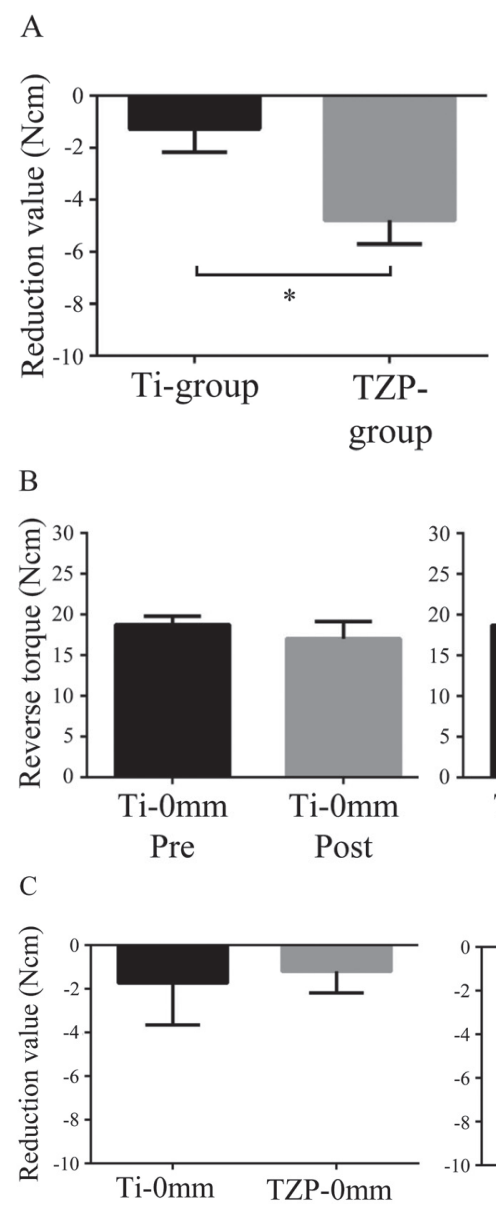

and quantitative evaluation of change in configuration comparing the influence of loading conditions in the TZP group were done to Student's $t$ test. GraphPad Prism (Version 6.05; GraphPad Software, San Diego, CA, USA) was used for the statistical analyses.

\section{RESULTS}

\section{Reverse torque}

In all specimens, the reverse torque values before cyclic loading test reduced against the fastening torque value recommended by manufacturer. The reduction value of $4.78 \mathrm{Ncm}$ in the TZP group was significantly larger than that of $1.28 \mathrm{Ncm}$ in the Ti group $(p<0.05)$ (Fig. 9A). The reverse torque values before and after test are shown in Fig. 9B within the same groups and under the same loading conditions. In all specimens, the reverse torque reduced after test compared to before test, and significant differences were indicated in Ti-4mm and TZP-4mm. The reduction value was $1.73 \mathrm{Ncm}$ in Ti-0mm, $1.20 \mathrm{Ncm}$ in TZP-0mm, 2.21 Ncm in Ti-4mm and 1.57 Ncm in TZP$4 \mathrm{~mm}$, respectively. However no significant difference was indicated between the groups (Fig. 9C).

\section{Marginal gap}

Comparison in the marginal gap between the groups under the same loading conditions are shown in Fig. 10A. The marginal gap for Ti-pre was $0.30 \mu \mathrm{m}$, Ti-0mm and Ti-4mm were 0.36 and $0.40 \mu \mathrm{m}$, respectively. That of TZP-pre was $0.71 \mu \mathrm{m}$, TZP-0mm and TZP- $4 \mathrm{~mm}$ were 0.71 and $0.57 \mu \mathrm{m}$, respectively. In all conditions, the TZP group showed significantly greater values compared to

Fig. 9 (A) Comparison between the groups by the reduction values from the tightening torque value to the reverse torque before test. ( $\left.{ }^{*} p<0.05\right)$. (B) Comparison within the same groups and under the same loading conditions by the reverse torque values before and after test $\left({ }^{*} p<0.05\right)$. (C) Comparison between the groups after the same loading conditions by the values reduced from before to after test. 
A
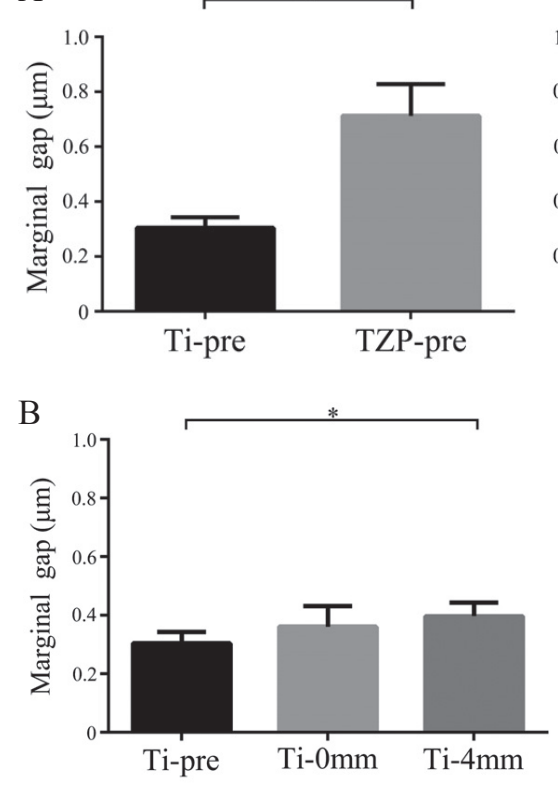
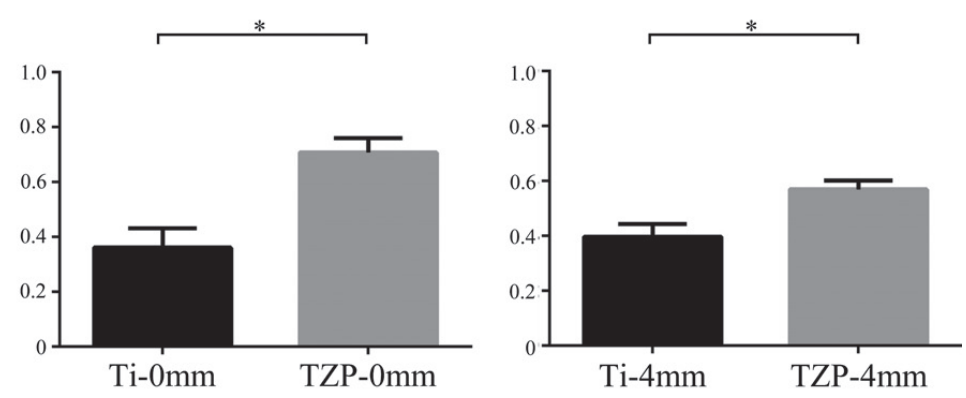

Fig. 10 (A) Marginal gap (Comparison between the groups under the same loading conditions) $\left({ }^{*} p<0.05\right)$.

(B) Marginal gap (Comparison between loading conditions within the same groups) ( $\left.{ }^{*} p<0.05\right)$.

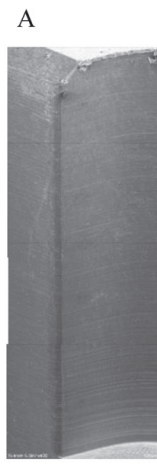

Ti-pre

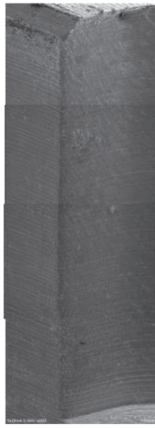

Ti-0mm

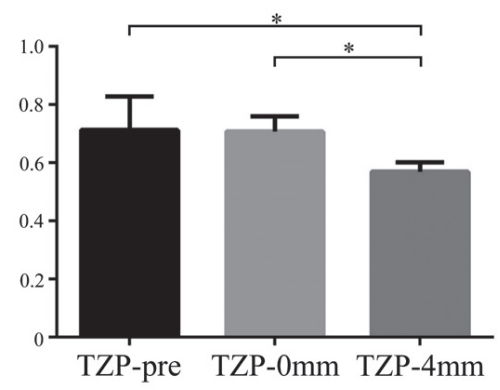

TZP-pre TZP-0mm TZP-4mm

Fig. 11 Representative SEM images in the Ti group.

(A) Implant body (B) Abutment.

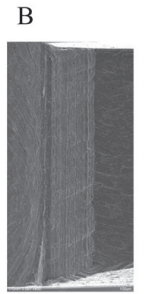

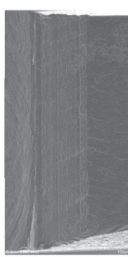

$\mathrm{Ti}-0 \mathrm{~mm}$

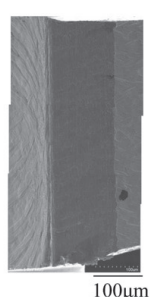

Ti-4mm

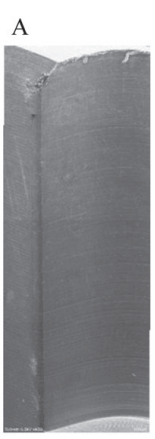

TZP-pre

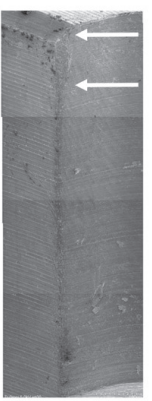

TZP-0mm

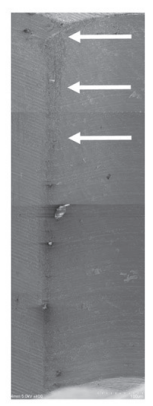

TZP-4mm
B

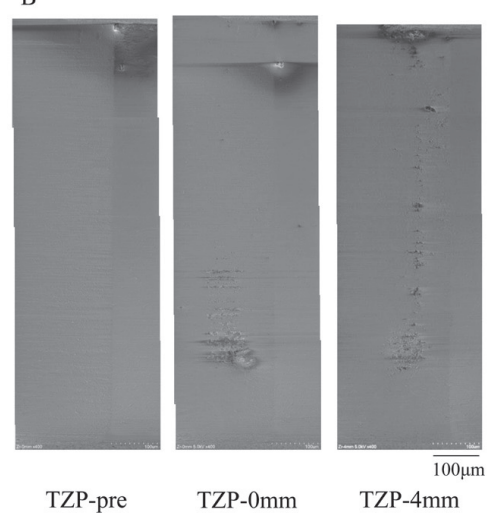

Fig. 12 Representative SEM images in the TZP group. (A) Implant body (B) Abutment. 
the Ti group $(p<0.05)$.

Comparison in the marginal gap between loading conditions within the same groups are shown in Fig. 10B. The Ti group showed larger marginal gap values after cyclic loading test and a significant difference was indicated between Ti-pre and Ti-4mm $(p<0.05)$. In the TZP group, the marginal gap was reduced after test. TZP-4mm showed a significantly lower value compared to TZP-pre and TZP-0mm $(p<0.05)$.

There was no significant difference in marginal gap between loading side and the non-loading side regardless of axial load or eccentric load in both Ti group and TZP group.

\section{Surface observation}

A representative SEM image of a specimen in each Ti and TZP group are shown in Figs. 11 and 12 respectively. In

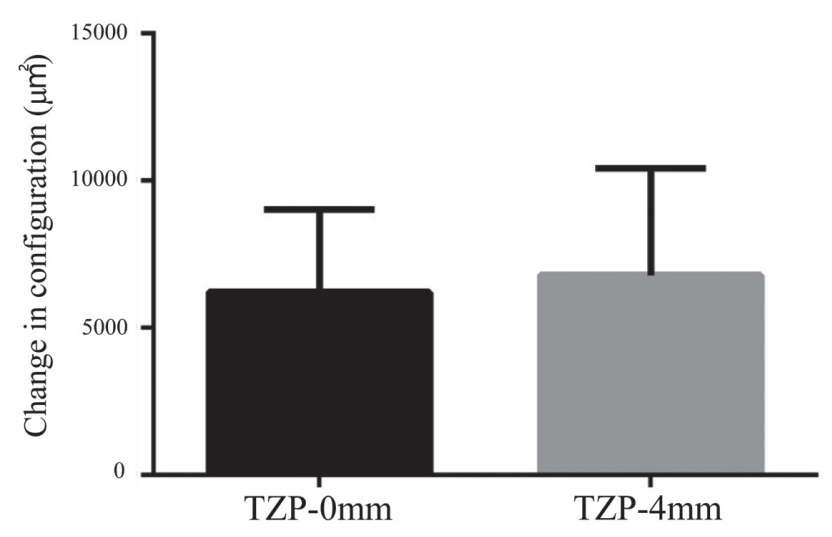

Fig. 13 Change in configuration of implant body in TZP group. the Ti group, no changes in configuration was observed in both the implant body and abutment. However, in the TZP group, changes in configuration, such as disappearance of the machining traces and formation of the smooth surface on the acute angle portion of the anti-rotation (arrow), was observed regardless of loading conditions. No changes were observed in the abutment of the TZP group.

Changes in configuration the anti-rotation of implant body (TZP group)

The Quantitative evaluation of changes in configuration of implant body for the TZP group are shown in Fig. 13. Values were $6,213.3 \mu \mathrm{m}^{2}$ for TZP-0mm and $6,790.8$ $\mu \mathrm{m}^{2}$ for TZP-4mm without differences between loading conditions.

\section{Elemental analysis of TZP group}

Results of EPMA analysis in the anti-rotation of abutment for TZP group are shown in Fig. 14. Titanium was detected on the anti-rotation regardless of loading conditions.

\section{DISCUSSION}

In the present study, cyclic loading test was conducted according to ISO $14801^{22)}$. Since this test can reproduce masticatory function while considering factors such as time and environment, reports indicate that this in-vitro test closely resembles clinical situations ${ }^{11)}$. Since the mesiodistal diameter is approximately 8 to $9 \mathrm{~mm}$ for the maxillary central incisor and $7.5 \mathrm{~mm}$ for the maxillary canine teeth ${ }^{25}$, a point $4 \mathrm{~mm}$ deviated from the axial load point in this study can be sufficiently assumed in clinical situation. The load cycles was set to $1 \times 10^{6}$ times
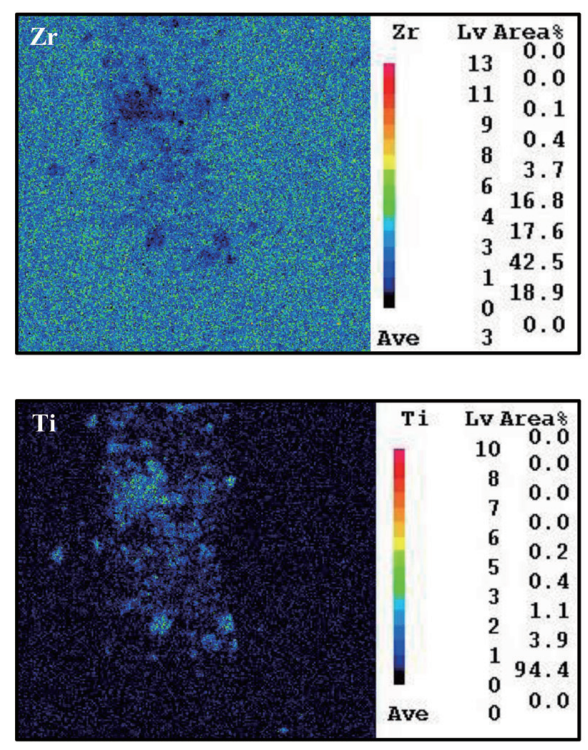

Fig. 14 Elemental analysis of abutment anti-rotation in TZP group. 
and which was equivalent to approximately 40 months of prosthesis use in the oral cavity and which strength fracture significantly decline ${ }^{2,11,26}$. The load was set to $300 \mathrm{~N}$ after considering that the maximum occlusal force of the incisal region was reported to be $299 \mathrm{~N}^{21}$. The test was carried out in distilled water at $37^{\circ} \mathrm{C}$ reproducing the temperature in the oral cavity. From the above, it can be considered that this experiment was conducted by a method assuming clinical conditions.

\section{Reverse torque value}

In this experiment, we measured the reverse torque value which can be used to indirectly investigate screw loosening ${ }^{17,27)}$. In recent years, it was also reported that abutment screws using titanium abutments is easy to loose compared to zirconia abutments ${ }^{12)}$. Accordingly, further study is necessary to investigate the loosening mechanism of the abutment screw using titanium and zirconia abutment.

The tightening force of the screw starts to loosen immediately after tightening. Therefore, in this experiment, the reverse torque value was measured by the method that minimizes loosening immediately with reference to other papers ${ }^{2,23,24)}$. The manufacturer recommended fastening torque value of the abutment screw used in this experiment was tightened higher in the TZP group than in the Ti group. As a result, there was no difference between the groups in the reduction value of the reverse torque after cyclic loading (Fig. $9 \mathrm{C}$ ), whereas the reverse torque value of the TZP group before cyclic loading reduced compared to that of the $\mathrm{Ti}$ group before cyclic loading (Fig. 9A). Therefore, the TZP group was suggested to cause screw loosening compared to the Ti group.

Furthermore, it was elucidated in this study that the reverse torque value reduces after the eccentric loading regardless of the groups (Fig. 9B).

\section{Marginal gap}

Methods using optical microscopes other than SEM have been used to measure marginal gaps ${ }^{17,28)}$. However, light is reflected when observing metallic materials using an optical microscope and SEM generally yields more accurate results ${ }^{17}$. Therefore, the marginal gap was measured using SEM in this experiment.

According to past reports on internal butt joint systems, marginal gaps under no load ranged widely from 1.38 to $5.6 \mu \mathrm{m}^{29,30)}$. In these studies, measurement method involved the use of left and right ends of the connecting segment as ROI. Therefore, in this study the ROI was established as the central region of the implant body. Magnification was increased to high magnification (5,000 times) in order to carry out an investigation in more detail. As a result, it was possible to measure the marginal gap of the $\mathrm{Ti}$ group in the range of 0.14 to $0.71 \mu \mathrm{m}$. For these reasons, the measurement method for marginal gap in this experiment was considered reasonable.

The TZP group displayed larger marginal gap values than the Ti group under all loading conditions (Fig. 10A).
This is also consistent with past reports which used the external joint system ${ }^{18}$. Zirconia is said to undergo sintering contraction as it is machined as semi-sintered blocks; these blocks are later subjected to a complete sintering in a high temperature furnace. This shrinkage caused errors which were $\mu \mathrm{m}$ unit $^{31}$. Therefore, the marginal fit of zirconia abutments can be considered to be worse than that of the titanium abutment already at the stage of manufacturing. Reports indicate that bacteria exist in a size of about $0.2 \mu \mathrm{m}^{32}$. Results of this study indicated that bacteria penetrated through the marginal gap and micro-leakage occurred in both the Ti and TZP groups. Micro-leakage tended to increase as marginal gap were large and cause inflammation to the surrounding tissue of the implant ${ }^{33}$. This suggests that the marginal gap observed in the TZP group may spread inflammation on surrounding tissues of the implant compared to the Ti group.

Past reports on the marginal gap of titanium abutments with internal butt joint systems indicated that the marginal gaps increased after eccentric loading ${ }^{28)}$. This is consistent with results of this experiment. However, there are no reports on the marginal gap of zirconia abutments. Results of this study indicated that the marginal gap of the TZP group reduced after eccentric loading (Fig. 10B). The possible reason is considered as follows; in the Ti group, due to the ductility of titanium material, plastic deformation occurs in the shank part of the abutment that was inserted in the implant body by eccentric loading on the butt joint type ${ }^{2)}$, leading that the marginal gap was increased caused by eccentric loading. On the other hand, in the TZP group, TZP abutment with worse fit and less ductility had penetrated into the implant body leading that large marginal gap having on manufacturing fabrication stage was reduced by eccentric loading. Furthermore, marginal gap changes by eccentric loading

\section{Change in configuration}

According to a past report investigating the internal joint system, changes in configuration that occurred in the platform region of the implant body was observed on zirconia abutments after eccentric loading ${ }^{19}$. This result is consistent with the results of this study. However, only eccentric loading were evaluated and the study did not compare between different loading conditions. In addition, there has been no report using the side of the anti-rotation as ROI, where is considered that the implant body and abutment comes in most contact during loading. In this study, for the TZP group, changes in configuration that occurred in the anti-rotation of the implant body was observed after both axial and eccentric loading. However, no significant differences in values of configurational change were indicated between loading conditions (Fig. 13). Results of the elemental analysis in the anti-rotation of abutment in the TZP group demonstrated that titanium was detected regardless of loading conditions (Fig. 14). This study suggested that changes in configuration occurred in the implant body when zirconia abutment was used, and abrasion deposits 
of titanium adhered to the abutment. As a result, it can be considered that the changes in configuration could occur on the implant body regardless of loading conditions when zirconia abutment is used.

\section{Limitations of this study}

This study is limited because only the internal butt joint system was evaluated. In a past report, the comparison between external joint system and internal joint system (both butt joints) when titanium abutment was used was investigated ${ }^{2}$. Since the zirconia abutment was not used, the larger eccentric load, such as a point $8 \mathrm{~mm}$ deviated from the axial load point, was investigated. In that case, the reduction of the reverse torque value was large in the external joint system, and component damage was observed in the internal joint system with the distal shift in the loading position. Further investigations including differences in joint systems should be conducted in the future, and it is believed that the best joint systems should be established.

\section{CONCLUSIONS}

In this study, the effects of eccentric cyclic loading on implant components using the internal joint system with titanium abutments (Ti group) and zirconia abutments (TZP group) were evaluated. Within the limitations of this study only using the internal joint system with butt joint connection, the following conclusions can be drawn:

1. The reduction value of reverse torque in the TZP group was significantly larger than that in the $\mathrm{Ti}$ group.

2. The marginal gap for the TZP group was larger already at the stage of manufacturing, and the marginal gap for the Ti group increased and the marginal gap for the TZP group reduced after eccentric loading.

3. Changes in configuration occurred in the antirotation of the implant body for the TZP group.

These results suggested that the TZP group was clinically disadvantageous compared to the Ti group.

\section{CONFLICTS OF INTEREST}

None.

\section{REFERENCES}

1) Flanagan D. Diet and implant complications. J Oral Implantol 2016; 42: 305-310.

2) Sakamoto K, Homma S, Takanashi T, Takemoto S, Furuya $\mathrm{Y}$, Yoshinari $\mathrm{M}$, et al. Influence of eccentric cyclic loading on implant components: Comparison between external joint system and internal joint system. Dent Mater J 2016; 35: 929937.

3) Watanabe F, Hata Y, Komatsu S, Ramos TC, Fukuda H. Finite element analysis of the influence of implant inclination, loading position, and load direction on stress distribution. Odontology 2003; 91: 31-36.

4) Khraisat A. Stability of implant-abutment interface with a hexagon-mediated butt joint: failure mode and bending resistance. Clin Implant Dent Relat Res 2005; 7: 221-228.

5) Wadhwani CPK, Schoenbaum T, King KE, Chung KH. Techniques to optimize color esthetics, bonding, and periimplant tissue health with titanium implant abutments. Compend Contin Educ Dent 2018; 39: 110-119.

6) Pinheiro Tannure AL, Cunha AG, Borges Junior LA, da Silva Concílio LR, Claro Neves AC. Wear at the implant-abutment interface of zirconia abutments manufactured by three CAD/ CAM systems. Int J Oral Maxillofac Implants 2017; 32: 12411250.

7) Tsumita M, Kokubo Y, Kano T, Sasaki K. Effect of fatigue loading on the screw joint stability of zirconium abutment. $J$ Prosthodont Res 2013; 57: 219-223.

8) Glauser R, Sailer I, Wohlwend A, Studer S, Schibli M, Schärer P. Experimental zirconia abutments for implant-supported single-tooth restorations in esthetically demanding regions: 4-year results of a prospective clinical study. Int J Prosthodont 2004; 17: 285-290.

9) Döring K, Eisenmann E, Stiller M. Functional and esthetic considerations for single-tooth Ankylos implant-crowns: 8 years of clinical performance. J Oral Implantol 2004; 30: 198209.

10) Barwacz CA, Brogden KA, Stanford CM, Dawson DV, Recker EN, Blanchette D. Comparison of pro-inflammatory cytokines and bone metabolism mediators around titanium and zirconia dental implant abutments following a minimum of 6 months of clinical function. Clin Oral Implants Res 2015; 26: e35-41.

11) Coray R, Zeltner M, Özcan M. Fracture strength of implant abutments after fatigue testing: A systematic review and a meta-analysis. J Mech Behav Biomed Mater 2016; 62: 333346.

12) Pjetursson BE, Zarauz C, Strasding M, Sailer I, Zwahlen M, Zembic A. A systematic review of the influence of the implantabutment connection on the clinical outcomes of ceramic and metal implant abutments supporting fixed implant reconstructions. Clin Oral Implants Res 2018; 29 Suppl 1: 160-183.

13) Flanagan D. Bite force and dental implant treatment: A short review. Med Devices (Auckl) 2017; 10: 141-148.

14) Butignon LE, Basilio M de A, Pereira RDP, Arioli Filho JN. Influence of three types of abutments on preload values before and after cyclic loading with structural analysis by scanning electron microscopy. Int J Oral Maxillofac Implants 2013; 28: e161-170.

15) Delben JA, Gomes EA, Barão VAR, Assunção WG. Evaluation of the effect of retightening and mechanical cycling on preload maintenance of retention screws. Int $\mathrm{J}$ Oral Maxillofac Implants 2011; 26: 251-256.

16) Yüzügüllü B, Avci M. The implant-abutment interface of alumina and zirconia abutments. Clin Implant Dent Relat Res 2008; 10: 113-121.

17) Butignon LE, de Almeida Basílio M, Santo JS, Arioli Filho JN. Vertical misfit of single-implant abutments made from different materials under cyclic loading. Int J Oral Maxillofac Implants 2016; 31: 1017-1022.

18) Markarian RA, Galles DP, Gomes França FM. Scanning electron microscopy analysis of the adaptation of singleunit screw-retained computer-aided design/computer-aided manufacture abutments after mechanical cycling. Int $\mathrm{J}$ Oral Maxillofac Implants 2018; 33: 127-136.

19) Stimmelmayr M, Edelhoff D, Güth J, Erdelt K, Happe A, Beuer F. Wear at the titanium-titanium and the titaniumzirconia implant-abutment interface: a comparative in vitro study. Dent Mater 2012; 28: 1215-1220.

20) Nguyen $H Q$, Tan KB, Nicholls JI. Load fatigue performance of implant-ceramic abutment combinations. Int $\mathrm{J}$ Oral Maxillofac Implants 2009; 24: 636-646.

21) Gehrke P, Dhom G, Brunner J, Wolf D, Degidi M, Piattelli A. Zirconium implant abutments: fracture strength and 
influence of cyclic loading on retaining-screw loosening. Quintessence Int 200; 37: 19-26.

22) ISO 14801. Dentistry - Implants— Dynamic fatigue test for endosseous dental implants, 2007

23) Tsuge T, Hagiwara Y. Influence of lateral-oblique cyclic loading on abutment screw loosening of internal and external hexagon implants. Dent Mater J 2009; 28: 373-381.

24) Yao KT, Kao HC, Cheng CK, Fang HW, Yip SW, Hsu ML. The effect of clockwise and counterclockwise twisting moments on abutment screw loosening. Clin Oral Implants Res 2012; 23: 1181-1186.

25) Stanley J. Nelson. Wheeler's Dental Anatomy, Physiology and Occlusion. 10th ed. St Louis: Elsevier; 2015 p. 99, 129.

26) Sakaguchi RL, Powers JM. Craig's restorative dental materials. 13th ed. St Louis: Mosby; 2012 p. 88.

27) Hagiwara M, Ohashi N. A new tightening technique for threaded fasteners. J Offshore Mech Arct Eng 1992; III-B: 371-376.

28) Jesus Tavarez RR de, Bonachela WC, Xible AA. Effect of cyclic load on vertical misfit of prefabricated and cast implant single abutment. J Appl Oral Sci 2011; 19: 16-21.

29) Tsuge T, Hagiwara $Y$, Matsumura H. Marginal fit and microgaps of implant-abutment interface with internal antirotation configuration. Dent Mater J 2008; 27: 29-34.

30) Hamilton A, Judge RB, Palamara JE, Evans C. Evaluation of the fit of CAD/CAM abutments. Int J Prosthodont 2013; 26: 370-380.

31) de França DGB, Morais MHST, das Neves FD, Barbosa GAS. Influence of CAD/CAM on the fit accuracy of implantsupported zirconia and cobalt-chromium fixed dental prostheses. J Prosthet Dent 2015; 113: 22-28.

32) Smith NA, Turkyilmaz I. Evaluation of the sealing capability of implants to titanium and zirconia abutments against Porphyromonas gingivalis, Prevotella intermedia, and Fusobacterium nucleatum under different screw torque values. J Prosthet Dent 2014; 112: 561-567.

33) Mishra SK, Chowdhary R, Kumari S. Microleakage at the different implant abutment interface: a systematic review. J Clin Diagn Res 2017; 11: ZE10-ZE15. 